\title{
Critical Illness Stress-induced Immune Suppression
}

\author{
J.A. Carcillo
}

\section{Introduction}

Despite the use of Centers for Disease Control and Prevention (CDC) recommended practices to minimize infection risk, nosocomial sepsis and multiple organ failure (MOF) remain a leading cause of morbidity and mortality in critically ill patients. It is well documented that the use of immunosuppressant therapies dramatically increases this risk in patients with cancer, transplantation, and immunologic disease. Although immune monitoring has yet to be universally embraced, withdrawal of immunosuppressant therapies and use of immune restoration therapies is the standard of care when these patients develop sepsis. Critical illness stress can also induce a level of immunosuppression which is as life-threatening as is seen in the purposefully immunosuppressed patient. This chapter reviews the role of critical illness stress-induced immunosuppression in the development of nosocomial sepsis and MOF, and outlines clinical strategies which can be employed to maintain and restore immune function, and reduce morbidity and mortality in critically ill patients.

\section{The Healthy Immune System: How We Recognize and Kill Invasive Microbial Pathogens}

The immune system has soluble and cellular components. If one takes soluble serum or plasma and exposes it to microbes there will be a certain amount of microbial killing, also known as serumcidal activity. This is orchestrated by endogenous antimicrobial polypeptides, including defensins, lactoferrin, and bactericidal permeability increasing factor (BPI). The complement system is also a major contributor to serumcidal activiy. In the first 12 hours of infection, circulating mannose binding lectin (MBL) binds to the mannose residues on the microbial surface and activates complement killing. After 12 hours, circulating antibodies and C-reactive protein (CRP) orchestrate a second wave of complement-mediated microbicidal activity.

The cellular component of the immune response also has two waves which are coordinated (Figs. 1 and 2). The first phase is called the innate immune system, which is predominant for 24 to 72 hours. Similar to the MBL pathway, these cells recognize microbial pathogens by a pattern of glycoproteins on their cell surface, which are not seen in human or eukaryote cells. These cells include polymorphonuclear leukocytes (PMN), monocyte/macrophages, and macrophage/dendritic cells, all of which engulf and directly kill microbes through the production of oxygen radicals and tumor necrosis factor (TNF) (only macrophage/dendritic cells produce 


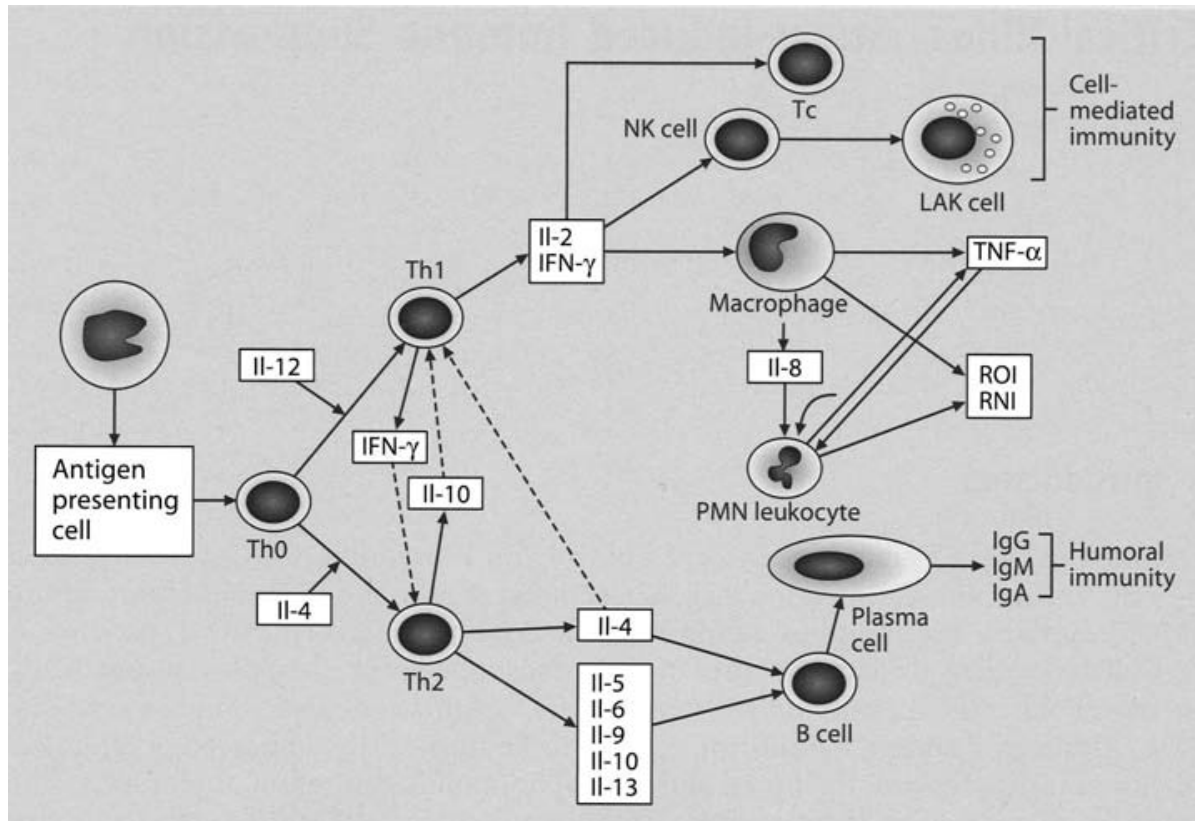

Fig. 1. The normal host immune response. IL: interleukin; IFN: interferon; TNF: tumor necrosis factor; NK: natural killer; ROI: reactive oxygen intermediates; RNI: reactive nitrogen intermediates

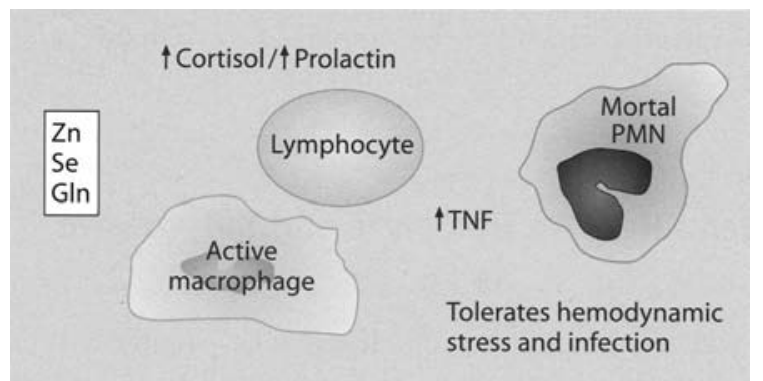

Fig. 2. The normal stress response maintains immune integrity

TNF). Once the bacteria are killed, the adherent PMN undergoes deactivation and apoptosis (programmed cell death) in response to macrophage/dendritic cell generated TNF. However, the macrophage/dendritic cell task is only beginning. These cells then process the antigenic peptides of the killed microbe and present them on the human leukocyte antigen (HLA)-DR antigen on their cell surfaces. Circulating T-lymphocytes now recognize this presented antigen and initiate the adaptive immune response approximately 24 to 72 hours after initial invasion. According to the cytokine milieu at the point of recognition, the adaptive immune response can go in one of two directions: the so called Th1 response or the Th2 response with the most effective host immune response being one that is well balanced between the two.

The Th1 response is more likely in the presence of interferon (IFN)- $\gamma$ and interleukin (IL)-2. During this process, the adaptive immune response is dedicated to 
more efficient killing. In the presence of viral infection, CD8 cytotoxic $\mathrm{T}$ cells and natural killer cells are recruited and activated to cell-mediated killing. In the presence of bacterial infection, macrophages call in more PMN leukocytes through secretion of IL-8, and also continue to efficiently kill with greater generation of TNF$\alpha$ and nitric oxide (NO)/oxygen radicals. In the presence of fungal infection, cytotoxic $T$ cells, natural killer cells, PMNs, and macrophages are all called upon for their fungicidal mission.

The Th2 response is more likely in the presence of IL-4, IL-6, and IL-10 and represents the predominant host response to parasitic infection. During this process, the Th1 response is dampened and B lymphocytes are produced from plasma cells. When Th1 and Th2 are balanced, these B cells participate in antibody production, which both enhances antibody-dependent, complement-mediated killing, and opsonizes encapsulated bacteria so they can be more efficiently phagocytosed by the reticuloendothelial system (macrophage/dendritic cells) in the spleen. However, when the Th2 response is predominant, antibodies are made but are ineffective because dendritic cells and macrophages cannot kill and process even antibodycoated organisms. A balanced Th1-Th2 response is the hallmark of effective and healthy immune function.

\section{How Can the Clinician Manipulate Immunosuppressant Therapies so they do not Prevent the Immune System from Recognizing and Killing Invasive Microbes and Cause Death from Sepsis?}

A number of immunosuppressant agents are commonly used with the purpose to kill or inactivate immune cells (Fig. 3). These include, among others, dexamethasone and other steroids, chemotherapy agents, irradiation, monoclonal and polyclonal antibodies, calcineurin inhibitors, and nucleoside analogs. Dexamethasone is among the most potent of all immunosuppressants. It is popular because it induces apoptosis (programmed cell death) in lymphocytes and malignant cells, rapidly reducing tumor size. It is also used widely for its profound anti-inflammatory and anti-TNF effects in the short term treatment of croup. However, although short term use is therapeutic, long term use promotes pseudomonas sepsis. Indeed, the experimental model of fatal pseudomonas pneumonia requires long term treatment with dexamethasone followed by pseudomonas inoculation [1]. Other steroids, including prednisone, methylprednisone, and hydrocortisone, do not share this degree of immunosuppressive effect $[2,3]$. Chemotherapy is generally directed to killing of rapidly dividing cells, and since immune cells are rapidly dividing, they are inadvertently killed. Chemotherapy for leukemias directly targets killing of either lymphocytes for lymphocytic leukemia, or PMN cells for acute myelogenous leukemia. These agents induce life-threatening neutropenia. Irradiation is commonly used to kill tumors and is also the mainstay for ablation of recipient bone marrow prior to bone marrow transplantation. Irradiation induces lymphocyte apoptosis. When resident macrophages/dendritic cells ingest these apoptotic lymphocytes they are deactivated and no longer able to recognize, phagocytose, or kill microbial invaders [4]. They are also unable to present antigenic peptides on HLA-DR molecules resulting in incapacitation of both the innate and adaptive immune systems. Monoclonal antibodies to lymphocytes are commonly used in solid organ transplant patients. T-cell antibodies, such as OK T3 or ATGAM, result in T cell lysis and depletion. However, unlike radiation or dexamethasone which cause death by apotosis, the mode of 


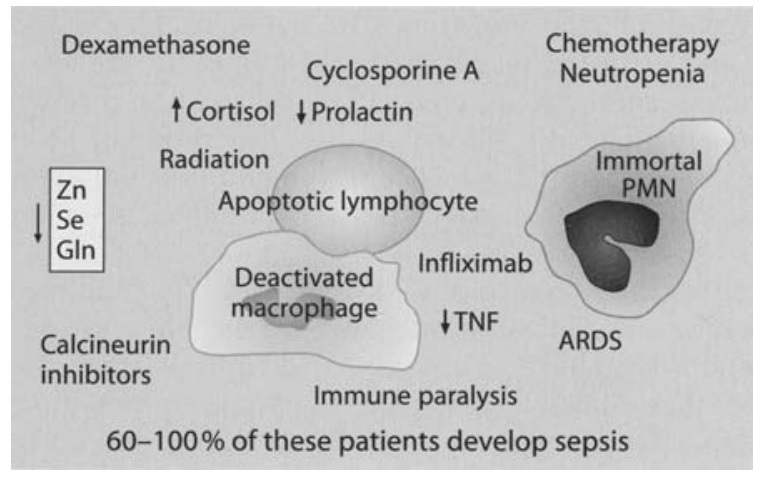

Fig. 3. Immunosuppressants can inhibit the ability to kill infection.

Table 1. Rational immune phenotype-directed therapeutic strategies in patients with critical illness stressinduced immunosuppression

\begin{tabular}{|c|c|}
\hline Immunophenotype thresholds & Therapeutic approach \\
\hline Absolute neutrophil count $<500$ cells $/ \mathrm{mm}^{3}$ & $\begin{array}{l}\text { a) Stop chemotherapy } \\
\text { b) Administer empiric antimicrobial therapy for } \\
\text { neutropenic fever; } \\
\text { c) Administer G-CSF, GM-CSF, or WBC infusion for } \\
\text { neutropenic sepsis }\end{array}$ \\
\hline Absolute lymphocyte count $<1,000$ cells $/ \mathrm{mm}^{3}$ & $\begin{array}{l}\text { a) Stop dexamethasone, dopamine, cyclosporine A } \\
\text { b) Administer prophylactic/empiric anti-viral, anti } \\
\text { fungal therapies } \\
\text { c) Replenish zinc, selenium, glutamine }\end{array}$ \\
\hline $\begin{array}{l}\text { Hypogammaglobulinemia } \\
\text { (lgG <500 mg/dl) }\end{array}$ & a) Give IVIG q three weeks \\
\hline $\begin{array}{l}\text { Monocyte deactivation - } \\
\text { HLA-DR }<30 \% \text { or } 8,000 \text { to } 12,000 \text { molecules/cell; } \\
\text { Whole blood TNF- } \alpha \text { response to LPS }<200 \mathrm{pg} / \mathrm{ml}\end{array}$ & $\begin{array}{l}\text { a) Stop dopamine, dexamethasone, calcineurin } \\
\text { inhibitors } \\
\text { b) Replenish zinc, selenium, glutamine } \\
\text { d) Apply appropriate antibiotic therapy and } \\
\text { remove the nidus of infection } \\
\text { e) Give GM-CSF } 125 \mu \mathrm{g} / \mathrm{m}^{2} \text { daily over } 12 \text { hours }\end{array}$ \\
\hline
\end{tabular}

TNF: tumor necrosis factor; GM-CSF: granulocyte-macrophage colony-stimulating factor; WBC: white blood cell; LPS: lipopolysaccharide; G-CSF: granulocyte colony-stimulating factor

death caused by these lytic therapies includes complement mediated necrosis. When macrophages/dendritic cells ingest these necrotic lymphocytes they remain active. Although the adaptive immune system is impaired, the innate immune system remains intact. B-cell antibodies are used to kill B-cell tumors caused by post-transplant lymphoproliferative disease or lymphoma. Anti-nucleoside analogs, by substituting for functional nucleoside building blocks, prevent DNA synthesis and lymphocyte proliferation in cancer and solid organ transplantation. The calcineurin inhibitors are mainstay immunosupressants in solid organ and bone marrow transplant patients. These agents mediate their effects by inducing a predominant Th2 state which inadvertently prevents Th1-mediated microbial phagocytosis, killing, processing, and presentation to the adaptive immune system. 
Fig. 4. Immune recovery with immunosuppressant withdrawal

Fig. 5. Lympholytic therapy is a novel strategy to maintain macrophage
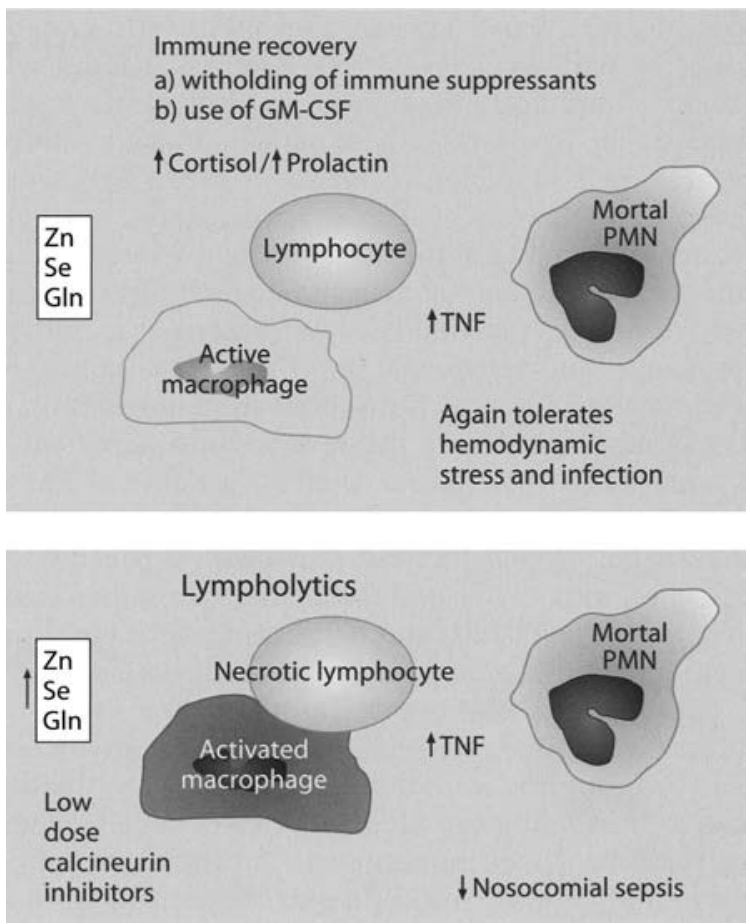

Immune phenotyping, although incompletely applied, has identified threshold values below which these immunosuppressants lead to life-threatening immunosuppression-induced nosocomial infection and sepsis (Table 1). An absolute neutrophil count less than 500 cells $/ \mathrm{mm}^{3}$ is associated with the development of sepsis. An absolute lymphocyte count $<1,000$ cells $/ \mathrm{mm}^{3}$ for more than three days is associated with a five-fold increased adjusted odds ratio for developing nosocomial sepsis [5]. If present more than seven days, there is a six-fold increased adjusted odds ratio for development of MOF and death. Immunoglobulin G levels $<500 \mathrm{mg} / \mathrm{dl}$ are also associated with secondary infection and sepsis. Prolonged monocyte deactivation for more than five days, also known as immunoparalysis, is associated with a six-fold increased adjusted odds ratio for nosocomial sepsis, MOF, and death [6]. The threshold for monocyte deactivation is defined by several functional tests including an HLA-DR expression $<30 \%$ of normal, or $<8,000-12,000$ molecules per monocyte cell, or a whole blood TNF- $\alpha$ response of $<200 \mathrm{pg} / \mathrm{ml}$ to lipopolysaccharide (LPS) stimulation. Specific therapeutic strategies utilized to prevent and treat nosocomial sepsis in special immunosuppressed patient populations are directed to restoration of immune function above these critical thresholds (Figs 4 and 5).

The most common critical threshold crossed in cancer patients is severe neutropenia. The American College of Oncology recommends the prophylactic use of growth factors including granulocyte colony-stimulating factor (G-CSF) or granulocyte/macrophage colony-stimulating factor (GM-CSF) to prevent neutropenia in patients who have previously had a $40 \%$ incidence of neutropenic fever after chemotherapy [7]. They also consider the use of G-CSF or GM-CSF reasonable therapy in patients with neutropenic sepsis. White blood cell transfusions are reserved for 
patients with sepsis who are unresponsive to growth factors. Other standard care in patients with neutropenic fever/sepsis includes withdrawal of chemotherapy and empiric antibitotic treatment for neutropenic fever. Antifungals are added if fever persists for five days. Cancer patients treated with prolonged courses of dexamethasone have a very high incidence of sepsis and sepsis-related death. Transition to a steroid regimen which uses prednisone, methylprednisone, or hydrocortisone completely reduces this risk likely by preventing lymphocyte apoptosis-related monocyte deactivation and immune paralysis. Bone marrow transplant patients suffer from pancytopenia. Because prolonged lymphopenia is the rule, anti-viral, antiprotozoal, and anti-fungal prophylaxis is administered daily. For patients with hypogammaglobulinemia, intravenous immunoglobulin (IVIG) is also administered on a tri-weekly basis. Bone marrow transplant patients with acute respiratory distress syndrome (ARDS) have a complete absence of Th1 activity in their lungs at autopsy [8]. This state of immune paralysis leads to an inability to kill viral, bacterial, and fungal pathogens. Immune function can be restored in part in these patients by stopping dexamethasone (switching to another steroid for graft versus host disease [GVHD] prophylaxis) and by titrating calcineurin inhibitor therapy such that monocyte function is above the critical threshold.

The field of solid organ transplantation has drastically changed its immunosuppressant regimens since its earliest days. Initially plagued by rejection when steroids and azathioprine were the therapy of choice, the field was boosted by the arrival of calcineurin inhibitors. Grafts no longer suffered acute rejection with implementation of steroid/calcineurin regimens, but patients commonly died from nosocomial sepsis and MOF. Volk and colleagues demonstrated that withholding steroids and calcineurin inhibitors from solid organ transplant patients with HLA-DR expression $<30 \%$ and sepsis resulted in near $100 \%$ survival without organ rejection, compared to $20 \%$ survival if immunosuppression was not withheld [9]. There are now over 300 publications on immunosuppression withdrawal from patients with solid organ transplantation as a strategy to induce immune tolerance. A popular regimen of immunosuppression today uses lytic therapies, such as OK T3 or ATGAM, which deplete lymphocytes through necrosis, not apoptosis, sparing steroid use and maintaining monocyte function above the critical threshold of immune paralysis.

Immunosuppressive/anti-proliferative regimens are also used in patients to shrink hemangiomas. Dexamethasone should not be used long term because of the risk of fatal pseudomonas infection. IFN $\alpha$ has been reported to be effective in this disease. Lymphomas caused by Epstein-Barr virus-mediated transformation with or without transplantation are treated with B cell monoclonal antibodies; however, IVIG must be administered for ensuant hypogammaglobulinemia.

The need for hypogammaglobulinemia treatment is also present in patients with systemic lupus erythematosis treated with B-cell lytic therapies. Macrophage activation syndrome in patients with rheumatologic disease is treated with apoptotic agents, such as dexamethasone, as well as with calcineurin inhibitors. The same principles as above should be applied to these patients. Dexamethasone should be reserved for short term use only and calcineurin inhibitors should be titrated to maintain monocyte/macrophage function above its critical functional threshold. 
Fig. 6. Critical illness stress-induced immunosuppression (CRISIS)

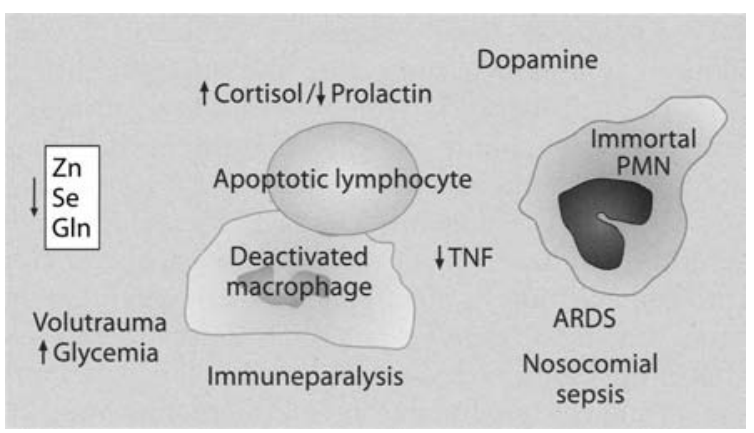

\section{Critical IIIness Stress-induced Immunosuppression}

Critical illness stress can induce prolonged lymphopenia and monocyte deactivation even when purposeful use of immunosuppressant therapies is absent. When stress induced immunosuppression is too great for too long the patient develops nosocomial sepsis, MOF, and death. The remainder of this chapter discusses clinical strategies which can be used to prevent and reverse critical illness stress-induced immunosuppression (Fig. 6).

A healthy patient subjected to surgical stress during a dental procedure or uncomplicated surgery will mount a hypothalamic/pituitary gland-mediated stress response with increased corticotropin releasing hormone/adrenocorticotropic hormone (CRH/ACTH) secretion and then adrenal gland cortisol production. This is matched by the increased production of the counter-regulatory hormone, prolactin, from the pituitary gland. The $\mathrm{CRH} / \mathrm{ACTH}$ and cortisol hormones maintain cardiovascular homeostasis and dampen inflammation. However, if left unopposed, ACTH/ $\mathrm{CRH} /$ cortisol also induce apoptosis of lymphocytes. Prolactin, through stimulation of $\mathrm{Bcl}_{2}$, inhibits this lymphocyte apoptotic effect of ACTH and cortisol. Nutrition is also important. Zinc, selenium, and glutamine are all necessary for control of lymphocyte apoptosis.

Critically ill neonates, children, and adults who die from nosocomial sepsis and MOF do so with prolonged lymphopenia (absolute lymphocyte count $<1,000>7$ days) and lymphoid organ depletion. At autopsy these patients have apoptosis-mediated depletion of B-cells from lymph nodes and spleen, $T$ cells from thymus, and dendritic cells from spleen [5]. Clinical factors associated with lymphoid depletion in these patients include use of synthetic steroids such as dexamethasone; zinc, glutamine, and/or selenium deficiency; and hypoprolactinemia. Zinc, glutamine, and selenium deficiency commonly occur in critical illness because these nutrients are consumed at higher rates during stress and commonly under dosed during parenteral and enteral nutritional support. Hypoprolactinemia can occur as part of the pituitary dysfunction syndrome of critical illness but can also be induced iatrogenically. Dopamine infusion stimulates the $\mathrm{DA}_{2}$ receptor, which prevents prolactin secretion by the pituitary gland, and cyclosporine $\mathrm{A}$ is a prolactin receptor antagonist. Hotchkiss and Nicholson have shown that caspase inhibitors prevent lymphopenia, reduce bacterial counts, and improve survival in the cecal ligation and puncture (CLP) model of experimental sepsis [10]. Chaudry and colleagues expanded upon this observation showing that treatment with prolactin or metoclopramide (a 
$\mathrm{DA}_{2}$ antagonist which increases prolactin levels) reverses hemorrhagic shock induced lymphocyte apoptosis and susceptibility to CLP sepsis-induced mortality [11]. Clinical therapies which can be used to reverse or prevent lymphopenia and lymphoid depletion in critically ill humans include provision of adequate zinc, glutamine, and selenium; withdrawal of dexamethasone, dopamine, and cyclosporine A; and use of $\mathrm{DA}_{2}$ antagonists, such as metoclopramide or haloperidol. In this regard, several trials have shown reductions in nosocomial infection in critically ill patients with the addition of zinc, glutamine, or selenium supplements. Norepinephrine has been shown to improve outcomes compared to dopamine [12], and a before and after study showed reduced nosocomial sepsis mortality when dexamethasone was substituted by prednisone or methylprednisone [2]. A single center study demonstrated an associated reduction in mortality in critically ill mechanically ventilated patients treated with haloperidol [13]. Two randomized controlled trials are underway: a single center study of haloperidol in mechanically ventilated adult patients, and a multiple center study of zinc [14], glutamine, selenium [15], and metoclopramide in critically ill pediatric patients.

Critical illness-induced lymphocyte apoptosis does not only result in lymphoid depletion, it also causes monocyte deactivation and immune paralysis. When macrophages are fed lymphocytes killed by irradiation (apoptotic lymphocytes) in vitro they become deactivated and have a diminished capacity to produce a TNF- $\alpha$ response to LPS. When macrophages are fed lymphocytes killed by freeze thaw (necrotic lymphocytes) they become activated and are able to produce TNF- $\alpha$ in response to LPS. Hotchkiss and colleagues have shown that adoptive transfer of apoptotic (irradiated) splenocytes sensitizes rats to CLP sepsis-induced mortality, while adoptive transfer of necrotic (freeze thaw) splenocytes protects rats to CLP sepsis induced mortality [16]. The effect is mediated through an IFN $\gamma$-Th1 mechanism. IFN $\gamma$ is decreased in apoptotic splenocytes and increased in necrotic splenocyte-treated subjects, and treatment with IFN $\gamma$ inhibition ablates the protective effect.

Prolonged monocyte deactivation and immune paralysis induced by lymphocyte apoptosis can also contribute to the development of ARDS. Although TNF- $\alpha$ does not induce apoptosis in circulating or non-adherent PMN leukocytes, it is needed for apoptosis of adherent PMN leukocytes [17]. When monocyte deactivation results after phagocytosis of apoptotic lymphocytes, its production of TNF- $\alpha$ is dramatically reduced. When the Thl mediated TNF- $\alpha$ response is absent, adherent PMN leukocytes will not apoptose. The hallmark of ARDS is lack of apoptosis of adherent lung PMN leukocytes. To test this possibility, Presneill and colleagues randomized patients with ARDS to GM-CSF therapy. GM-CSF effectively increases the TNF- $\alpha$ Th1 response in deactivated macrophages in the absence of apoptotic lymphocytes, dexamethasone, and calcineurin inhibitors. In this study, no information was given on the presence or absence of these factors. Nevertheless, GM-CSF reduced lung PMN cells, supporting a role for TNF- $\alpha$ in facilitating adherent PMN leukocytes during ARDS [18].

Mechanical stress caused by volutrauma from overzealous mechanical ventilation also leads to a predominant Th2 state with prolonged monocyte deactivation, immunoparalysis, and continued ARDS. Healthy children subjected to large tidal volumes of $12 \mathrm{ml} / \mathrm{kg}$ during elective surgery have been documented to have increased circulating levels of IL- 6 and IL-10 with reduced monocyte production of TNF- $\alpha$. Healthy children subjected to physiologic tidal volumes of $6-8 \mathrm{ml} / \mathrm{kg}$ have no increase in IL- 6 or IL-10 levels and maintain normal monocyte TNF- $\alpha$ produc- 
tion [19]. Adults with ARDS receiving larger tidal volumes show similar relationships with increased systemic IL- 6 and IL-10, reduced monocyte TNF- $\alpha$ production, and immune paralysis [20]. In this regard, mechanical ventilation with effective tidal volumes of $6-8 \mathrm{ml} / \mathrm{kg}$ reduces mortality from ARDS [21].

Endotoxemia and sepsis also lead to prolonged monocyte deactivation and immunoparalysis in critically ill patients. Experimental animals and humans subjected to endotoxin or sepsis challenge, develop a reduced or down regulated monocyte/macrophage derived TNF- $\alpha$ response to LPS. The mechanism of this 'endotoxin tolerance' appears to be driven in part by an IL-10/IL-6 - Th2 milieu which subsequently inhibits the Th1 response. This state of 'endotoxin tolerance', initially thought to be protective, is now known to harmfully increase susceptibility to sepsis-induced death. Experiments show that Th1 dominant mice are more resistant to sepsis than Th2 dominant mice [22]. Treatment with flt-3 ligand, the dendritic cell growth factor, reverses endotoxin tolerance, restores the TNF- $\alpha$ response to LPS, and reduces susceptibility to sepsis mortality [23]; so too does treatment with GMCSF and IFNy $[24,25]$.

The term 'endotoxin tolerance' is not used in humans. Instead patients with sepsis or endotoxemia who develop a TNF- $\alpha$ response to LPS of $<200 \mathrm{pg} / \mathrm{ml}$ and/or decreased monocyte HLA-DR expression $<30 \%$ or $8,000-12,000$ molecules per cell are said to have monocyte deactivation and immunoparalysis. General surgery patients with immunoparalysis have an $80 \%$ mortality with sepsis, compared to only $12 \%$ mortality in those who have monocyte function above this threshold [9]. In vitro studies show that two drugs, GM-CSF and IFN $\gamma$, can reverse endotoxin- or sepsis-induced monocyte deactivation in humans [24]. One case series has demonstrated reversal of immunoparalysis with IFN therapy [26]; however, IFN therapy has greater toxicity than GM-CSF therapy. A randomized controlled trial using a three day course of low-dose GM-CSF demonstrated improved monocyte HLA-DR expression with improved cure rates and resolution of sepsis [27]. Studies designed to evaluate the effectiveness of GM-CSF in patients with sepsis, ARDS, or MOF can provide confusing results if not done with immune phenotyping as well as proper source control. GM-CSF is not effective in reversing immune paralysis if monocytes/macrophages continue to phagocytose apoptotic lymphocytes, or to be immunosuppressed by exogenous immunosuppressant therapy. Hence immunosuppressants, dexamethasone, and dopamine should all be withheld during GM-CSF therapy. GM-CSF will also not be helpful in a patient with sepsis who does not have immune paralysis but instead suffers from failure to remove the nidus of infection due to lack of appropriate antibiotic therapy or surgical drainage. For example, in the activated protein $C$ trial there was no benefit observed in surgical sepsis; however, the mortality rates in those with proper nidus removal and source control was $1 \%$ compared to $92 \%$ without proper source control [28]. Patients can have monocyte function above critical threshold levels and still die due to lack of removal of the source of sepsis.

Trauma patients also develop stress induced immunosuppression. Lymphopenia is a marker of severity of illness and mortality in trauma and should be addressed with nutritional attention to zinc, selenium, and glutamine status as well as avoidance of dopamine. In patients with severe head injury, the use of pentobarbital, hypothermia, and hypertonic saline all reduce PMN leukocyte function. These therapies should be limited, particularly in patients who develop nosocomial sepsis. Surgical intervention for refractory intracranial hypertension can be preferred to this immunosuppressive regimen in selected patients. Prolonged narcotic use can also decrease PMN leukocyte function and induce monocyte deactivation [29]. Use of 
low dose naloxone can be effective in reducing opioid tolerance and potentially reduces these immunosuppressant effects. Hyperglycemia can also impair innate and adaptive immune function. A randomized controlled trial showed that when daily glucose requirements were met (D10 infusion at maintenance fluid dosage) and hyperglycemia was controlled with insulin, surgical patients experienced less nosocomial sepsis, MOF, and mortality [30].

\section{Conclusion}

Despite implementation of CDC recommendations for prevention of infection, nosocomial sepsis and MOF remain the most common acquired causes of death and morbidity in critically ill patients. The role of immune dysfunction in this process is evidenced by an increased risk of nosocomial sepsis in patients hospitalized with immune deficiency and/or receiving immunosuppressant therapies [31]. Four iatrogenic immune deficiency thresholds appear important in predicting and reversing risk of nosocomial sepsis: 1) neutropenia, defined as an absolute neutrophil count $<500$ cells $/ \mathrm{mm}^{3}$; 2) monocyte deactivation or immune paralysis defined by monocyte HLA DR expression $<30 \%$ or $<8000-12000$ molecules per cell or whole blood TNF- $\alpha$ response to LPS of $<200 \mathrm{pg} / \mathrm{ml}$; 3) lymphopenia, defined as an absolute lymphocyte count $<1,000$ cells $/ \mathrm{mm}^{3}$; and 4) hypogammaglobulinemia, defined as an IgG level $<500 \mathrm{mg} / \mathrm{dl}$. Therapeutic strategies in these populations include: a) empiric and prophylactic use of antibiotics, anti-virals, anti-protozoals, and antifungals; b) withdrawal of immunosuppressant therapy; c) administration of G-CSF, GM-CSF, and IVIG; and d) use of lympholytic rather than lymphocyte apoptotic agents to induce immune tolerance.

Critical illness stress-induced immunosuppression similarly causes patients to reach critical threshold levels of lymphopenia, monocyte deactivation, and immune paralysis and to develop nosocomial sepsis and MOF. Lymphocyte apoptosis and lymphoid depletion occur when the CRH/ACTH/cortisol axis induces programmed cell death in the presence of zinc, selenium, and glutamine deficiency, and/or hypoprolactinemia caused by pituitary dysfunction or dopamine infusion. Immunoparalysis can be caused by macrophage ingestion of apoptotic lymphocytes, or by mechanical ventilation-related volutrauma or endotoxin/sepsis induced Th2-mediated inhibition of macrophage TNF- $\alpha$ production. Surgical and traumatic stress similarly induce lymphopenia and Th2 mediated immunoparalysis. Randomized controlled trials, and before and after studies suggest that nosocomial sepsis can be reduced and survival outcome improved with zinc, selenium, and glutamine supplementation, dexamethasone and dopamine withdrawal, limitation of mechanical ventilator delivered tidal volumes to $6-8 \mathrm{~m} / \mathrm{kg}$, effective source control with appropriate antibiotics and nidus removal, and appropriate glucose delivery with insulin therapy directed to glycemic control. Once these tasks have been accomplished, GMCSF can permit further restoration of the Th1 response, reversing residual immune paralysis, and preventing/resolving nosocomial sepsis.

\section{References}

1. Satoh S, Oishi K, Iwagaki A, et al (2001) Dexamethasone impairs pulmonary defence against Pseudomonas Aeruginosa through suppressing iNOS gene expression and peroxynitrite production by mice Clin Exp Immunol 126:266-273 
2. Hurwitz CA, Silverman LB, Schorin MA, et al (2000) Substituting dexamethasone for prednisone complicates remission induction in children with acute lymphoblastic leukemia. Cancer 88:1964- 1999

3. Hirano T, Horigome A, Takatani M, Oka K (2001) Cortisone counteracts apoptosis inducing effect of cortisol in human peripheral blood mononuclear cells. Int Immunopharmacol $1: 2109-2115$

4. Green DR, Beere HM (2000) Apoptosis. Gone but not forgotten. Nature 405:28-29

5. Felmet KA, Hall MW, Clark RS, Jaffe R, Carcillo JA (2005) Prolonged lymphopenia, lymphoid depletion, and hypoprolactinemia in children with nososcomial sepsis and multiple organ failure. J Immunol 174:3765-3772

6. Volk HD, Reinke P, Docke WD (1999) Immunological monitoring of the inflammatory process: Which variables? When to assess? Eur J Surg Suppl (584):70-72

7. Smith TJ, Khatcheressian J, Lyman GH, et al (2006) 2006 update of recommendations for the use of white blood cell growth factors: an evidence based clinical pratcice guideline. J Clin Oncol 24:3187-3205

8. Sparrelid E, Emanuel F, Fehninger T, Andersson U, Andersson J (1997) Interstitial pneumonitis in bone marrow transplant recipients is associated with local production of TH2-type cytokines and lack of $\mathrm{T}$ cell mediated cytotoxicity. Transplantation 63:1782-1789

9. Volk HD, Reinke P, Krausch D, et al (1996) Monocyte deactivation - rationale for a new therapeutic strategy in sepsis. Intensive Care Med 22 (Suppl 4):S474-481

10. Hotchkiss RS Nicholson DW (2006) Apoptosis and caspases regulate death and inflammation in sepsis. Nat Rev Immunol 6:813-822

11. Chaudry IH, Samy TS, Schwacha MG, et al (2003) Endocrine targets in experimental shock. J Trauma 54 (Suppl 5):S118-125

12. Mullner M, Urbanek B, Havel C, Losert H, Waechter F, Gamper G (2004) Vasopressors for shock. Cochrane Database Syst Rev CD003709

13. Millbrandt EB, Kersten A, Kong L, et al (2005) Haloperidol use is associated with lower hospital mortality in mechanically ventilated patients. Crit Care Med 33:226-229

14. Brooks WA, Yunus M, Santosham M, et al (2004) Zinc for severe pneumonia in very young children: double blind placebo controlled trial. Lancet 363:1683-1688

15. Darlow BA, Austin NC (2003) Selenium supplementation to prevent short term morbidity in pre term neonates. Cochrane Database Syst Rev CD003312

16. Hotchkiss RS, Chang KC, Grayson MH, et al (2003) Adoptive transfer of apoptotic splenocytes worsens survival, whereas adoptive transfer of necrotic splenocytes improves survival in sepsis. Proc Natl Acad Sci USA 100:6724-6729

17. Avdi NJ, Nick JA, Whitlock BB, et al (2001) Tumor necrosis factor alpha activation of the cJun N-terminal kinase pathway in human neutrophils. Integrin involvement in a pathway leading from cytoplasmic tyrosine kinases apoptosis. J Biol Chem 276:2189-2199

18. Presneill JJ, Harris T, Stewart AG, Cade JF, Wilson JW (2002) A randomized phase II trial of granulocyte macrophage colony stimulating factor therapy in severe sepsis with respiratory function. Am J Respir Crit Care Med 166:138-142

19. Plotz FB, Vreugdenhil HA, Slutsky AS, et al (2002) Mechanical ventilation alters the immune response in children without lung pathology. Intensive Care Med 28:486-492

20. Ranieri VM, Suter PM, Tortorella C, et al (1999) Effect of mechanical ventilation on inflammatory mediators in patients with acute respirtaory distress syndrome: a randomized controlled trial. JAMA 282:54-61

21. The Acute Respiratory Distress Syndrome Network (2000) Ventilation with lower tidal volumes as compared with traditional tidal volumes for acute lung injury and the acute respiratory distress syndrome. $\mathrm{N}$ Engl J Med 342:1301-1308

22. Watanabe $H$, Numata $K$, Ito $T$, Takagi $K$, Matsukawa $A$ (2004) Innate immune response in TH1 and TH2 dominant mouse strains. Shock 22:460-468

23. Wysocka M, Montaner LJ, Karp CL (2005) Flt 3 ligand treatment reverses endotoxin tolerance related immunoparalysis. J Immunol 174:7396-7402

24. Randow F, Docke WD, Bundschuh DS, et al (1997) In vitro prevention and reversal of lipopolysaccharide desensitization by IFN gamma, IL-12, and granulocyte macrophage colony stimulating factor. J Immunol 158:2911-2918

25. Bundshuh DS, Barsig J, Hartung T, et al (1997) Granulocyte macrophage colony stimulating 
factor and IFN gamma restore the systemic TNF alpha response to endotoxin in liposacchariade desensitized mice. J Immunol 158:2862-2871

26. Docke WD, Randow F, Syrbe U, et al (1997) Monocyte deactivation in septic patients : retsortaion by IFN-gamma treatment. Nat Med 3:678-681

27. Rosenbloom AJ, Linden PK, Dottance A, et al (2005) Effect of granulocyte-monocyte colony stimulating factor therapy on leukocyte function and clearance of serious infection in nonneutropenic patients. Chest 127:2139-2150

28. Barie PS, Williams MD, McCollam JS, et al (2004) Benefit/risk profile of drotrecogin alfa (activated) in surgical patients with severe sepsis. Am J Surg 188:212-220

29. Greeneltch KM, Kelly-Welch AE, Shi Y, Keegan AD (2005) Chronic morphine treatment promotes specific TH2 cytokine production by nurine T cells in vitro via a Fas/Fas liganddependent mechanisms. J Immunol 175:4999-5005

30. van den Berghe G, Wouters $P$, Weekers $F$, et al (2001) Intensive insulin therapy in the critically ill patients. N Engl J Med 345:1359-1367

31. Williams MD, Braun LA, Cooper LM, et al (2004) Hospitalized cancer patients with severe sepsis: analysis of incidence, mortality, and associated costs of care. Crit Care 8:R291-298 